\title{
6. Fazit: Daten, Phänomene und Theorien
}

James Bogen und James Woodward bemängeln, dass die allermeisten zeitgenössischen Wissenschaftstheoretiker mit zweigliedrigen Modellen der wissenschaftlichen Praxis arbeiten, die ausschließlich zwischen Theorien und Beobachtungsergebnissen differenzieren. Diese Modelle berücksichtigen nicht, so der Vorwurf, dass es in der wissenschaftlichen Praxis einen Unterschied zwischen Daten und Phänomenen gebe. Eine angemessene Beschreibung der wissenschaftlichen Praxis müsse auf einem dreigliedrigen Wissenschaftsmodell beruhen, das die Elemente Daten, Phänomene und Theorien auseinander halte. Bogen und Woodward erläutern den Unterschied zwischen Daten und Phänomenen anhand zahlreicher Beispiele. Es erweist sich allerdings als schwierig, präzise anzugeben, worin genau er besteht. Insbesondere eine überzeugende Klärung der Frage, was naturwissenschaftliche Phänomene sind, fehlt in der derzeit vorliegenden Literatur. Ziel dieses Teils meiner Arbeit war es deshalb, die Bedeutung des Phänomenbegriffs zu klären, um darauf aufbauend eine tragfähige Interpretation der Daten-Phänomen-Unterscheidung zu entwickeln. Hierbei komme ich zu folgendem Ergebnis:

Phänomene sind diejenigen Sachverhalte, die in der wissenschaftlichen Praxis zwei wichtige Rollen spielen: Sie sind die Sachverhalte, die Wissenschaftler erklären wollen, und diejenigen, die sie als Belege für weitergehende Theorien anführen. Damit Phänomene diese Rollen übernehmen können, müssen Aussagen, die diese Phänomene beschreiben, im Lichte gängiger methodologischer Standards akzeptiert werden. Alle drei Begriffsmerkmale (Explandum- und Belegrolle sowie Akzeptanz) wurden in den vorhergehenden Kapiteln näher erläutert. Dass ich Phänomene dabei als Sachverhalte auffasse, ist eine eher pragmatisch begründete Entscheidung, die Einheitlichkeit gewährleistet und gut zu den drei Begriffsmerkmalen passt, sollte aber nicht als starke philosophische These gelesen werden. Die philosophischen Thesen, die ich hoffe begründet zu haben, betreffen vielmehr nur die Zuschreibung der drei genannten Begriffsmerkmale.

Dieses kontextuelle Verständnis des Phänomenbegriffs liefert die Grundlage dafür, wie Bogens und Woodwards Unterscheidung zwischen Daten und Phänomenen zu verstehen ist. Daten und Phänomene sind begrifflich verschieden und nicht, wie es zuerst nahe liegend erschien, über die unterschiedlichen Arten und Weisen unseres epistemischen Zugangs zu ihnen. Das Verhältnis von Phänomenbehauptungen und Theorien wiederum kann 
nur angemessen eingefangen werden, wenn man den Phänomenbegriff als kontextuellen Begriff auffasst. Phänomene sind die Forschungsgegenstände von Wissenschaftlern, auf sie richten sie ihre Erklärungsabsichten und auf sie wird sich berufen, um Theorien zu belegen. Theorien liefern Beschreibungen von Sachverhalten. Unterschiedliche Theorien und unterschiedliche Teilklassen der Aussagen einer Theorie können dabei unterschiedlich gut bestätigt sein und gemäß akzeptierten methodologischen Standards gut bestätigte theoretische Aussagen kommen als Phänomenbehauptungen in Frage. Diese begrifflichen Unterschiede zwischen Daten und Phänomenen werden, darin stimme ich Bogen und Woodward zu, in philosophischen Modellen der wissenschaftlichen Praxis in der Regel nicht beachtet. Eine möglichst angemessene Beschreibung der wissenschaftlichen Praxis sollte hingegen dreigliedrig sein, d.h. zwischen Daten, Phänomenen und Theorien unterscheiden. 